\title{
Shape-optimization of 2D hydrofoils using an Isogeometric BEM solver
}

\author{
K.V. Kostas ${ }^{\mathrm{a}, *}$, A.I. Ginnis ${ }^{\mathrm{b}}$, C.G. Politis ${ }^{\mathrm{c}}$, P.D. Kaklis ${ }^{\mathrm{d}, \mathrm{e}}$ \\ ${ }^{a}$ Department of Mechanical Engineering, Nazarbayev University \\ ${ }^{b}$ School of Naval Architecture \& Marine Engineering, National Technical University of Athens \\ ${ }^{c}$ Department of Naval Architecture, Technological Educational Institute of Athens \\ ${ }^{d}$ Department of Naval Architecture, Ocean and Marine Engineering, University of Strathclyde \\ ${ }^{e}$ EPICE AROMATH, Inria, Sophia Antipolis Méditerranée
}

\begin{abstract}
In this paper, an optimization procedure, based on an Isogeometric BEM solver for the potential flow, is developed and used for the shape optimization of hydrofoils. The formulation of the exterior potential-flow problem reduces to a Boundary-Integral Equation (BIE) for the associated velocity potential exploiting the null-pressure jump Kutta condition at the trailing edge. The numerical solution of the BIE is performed by an Isogeometric Boundary-Element Method (BEM) combining a generic B-splines parametric modeler for generating hydrofoil shapes, using a set of eight parameters, the very same basis of the geometric representation for representing the velocity potential and collocation at the Greville abscissas of the knot vector of the hydrofoil's B-splines representation. Furthermore, the optimization environment is developed based on the geometric parametric modeler for the hydrofoil, the Isogeometric BEM solver and an optimizer employing a controlled elitist genetic algorithm. Multi-objective hydrofoil shape optimization examples are demonstrated with respect to the criteria i) maximum lift coefficient and ii) minimum deviation of the hydrofoil area from a reference area.
\end{abstract}

Keywords: Isogeometric Analysis, NURBS, Potential Flows, Lifting Flows, Shape optimization

\section{Introduction}

Performance of many ship-hull types is dependent on the hydrodynamic properties of their keels, rudders, hydrofoils and similar appendages operating as lifting bodies. Therefore, the interaction of free-surface flows with lifting bodies constitutes an interesting problem, finding applications in the design of yachts and sailing boats and the performance of stabilizers, hydrofoils and similar devices. As a common feature, the functionality of these lifting appendages depends on their shape and even small variations of it may have significant impact on their performance. Fortunately, the current availability of computing power has allowed the employment of sophisticated Computational Fluid Dynamics solvers and their coupling with advanced Geometric Modeling techniques and Optimization strategies for the design optimization of both ship hull and lifting appendages. In this context, computational tools that represent exactly body shapes, securing that mesh generation is an error-free process provide designers and researchers with a great challenge. Moreover, the idea of bridging the gap between Computer-Aided Design (CAD) and Analysis by the introduction of IsoGeometric Analysis (IGA) by Hughes et al (2005) [1], see also Cottrell et al (2009) [2], which directly uses analysis suitable geometric models from the CAD representation, can be efficiently exploited, especially in problems of shape optimization. Shape optimization in the context of IGA has been presented in various works, as e.g., Wall et al (2008) [3], Nagy et al (2009) [4], Nguyen et al (2012) [5], Nortoft \& Gravesen (2013) [6], Qian \& Sigmund (2011) [7], Cho \& Ha (2009) [8], Lian et al (2013) [9], Qian (2010) [10], Li \& Qian (2011) [11], where NURBS

\footnotetext{
*Corresponding author: konstantinos.kostas@nu.edu.kz
} 
control points and/or weights have been used as design variables to control the boundary shape. On the other hand, in Kostas et al (2015) [12] and Ginnis et al (2013) [13], due to the shape complexity of the ship hull, a geometric parametric model using high-level parameters with a direct design meaning has been developed and used for optimization purposes in the context of IGA approach.

The main purpose of the present work is to develop an optimization procedure for the shape optimization of hydrofoils. This task is accomplished by combining three components: An optimization algorithm, an IGA-BEM solver for the potential flow outside the hydrofoil, presented in $\S 2$, and a hydrofoil geometric parametric model, presented in $\S 3.2$.

The formulation of the exterior potential-flow problem is reduced to a Boundary Integral Equation (BIE) for the associated velocity potential on the body boundary, exploiting the nullpressure jump Kutta condition at the trailing edge, Morino (1993) [14], Gennaretti et al (1998) [15]. Boundary element methods (BEM) in aero/hydrodynamics were established as main tools for the solution of flow problems around lifting and/or non-lifting bodies of arbitrary shape; see, e.g., Katz \& Plotkin (1991) [16], Paris \& Canas (1997) [17], Dragos (2003) [18]. Starting from the pioneering work by Hess \& Smith (1962) [19], where the 3D panel method based on source-sink distribution is presented for analyzing the flow around arbitrary non-lifting bodies, this approach has been further extended by Hess (1972) [20] for lifting flows and applied by Hess and Valarezo (1985) [21] for the simulation of a steadily translating and rotating propeller. Concentrating on marine propulsors, in the years to follow, various alternative formulations of the boundary element method have been applied for the solution of propeller related steady or unsteady problems. Most boundary element methods for lifting and propeller flows solve directly for the unknown velocity potential, a formulation more stable from the numerical point of view, see, e.g., Morino \& Kuo (1974) [22], Kinnas (1996) [23], although several alternatives exist, as for example the methods based on surface vorticity distributions developed by Belibassakis \& Politis $(1995,1998)$ [24], [25]. In the last period, higher-order BEM, based on B-splines or NURBS representations for the geometry and/or the solution, have been presented for the analysis of flow around marine propellers; see, e.g., Lee \& Kerwin (2003) [26], Kim et al (2007) [27], Gao \& Zou (2008) [28]. In the present work, the IsoGeometric Analysis - Boundary Element Method (IGA-BEM) initiated by Politis et al (2009) [29], for the solution of an exterior potential-flow problem without lift, is extended and applied for the corresponding problem with lift. The IsoGeometric Analysis concept, introduced by Hughes et al (2005) [1] in the context of Finite Element Method, was extended to the Boundary Element Method by various authors, see, e.g., Politis et al (2009) [29], Simpson et al (2012) [30], Scott et al (2013) [31], Belibassakis et al (2013) [32], Peake et al (2013) [33], Simpson et al (2014) [34], Ginnis et al (2014) [35]. The developed Isogeometric-BEM is based on a parametric NURBS representation of the hydrofoil and employs the very same basis of the geometry for representing the velocity potential. The Boundary Integral Equation is numerically solved by collocating at the Greville abscissas of the knot vector of the hydrofoil's parametric representation. Numerical error analysis of the Isogeometric-BEM, using h-refinement technique (knot insertion), is performed and compared with classical low-order panel methods.

As regards the geometric parametric model for the hydrofoil, it is constructed within Rhinoceros 3D using its VBscript-based programming language. This parametric model generates an instance of the hydrofoil as a closed cubic B-spline curve using a set of eight design parameters. The construction process is presented in detail in $§ 3.2$. The optimization loop is driven by an optimizer which uses a multi-objective optimization method based on a controlled elitist Genetic Algorithm (GA) favoring individuals that increase the diversity of the population; see $\S 3.1$. Finally, the optimization environment has been tested by optimizing the shape of a hydrofoil with respect to the following two criteria: maximum lift coefficient and minimum deviation from a reference cross-section area; see $\S 3.3$.

\section{Continuous and discrete formulation of the problem}

We consider a two-dimensional body whose boundary is $\partial \Omega_{B}$, as shown in Figure 1, moving with constant speed $\vec{U}_{B}$ in an ideal fluid of infinite extent. In a body-fixed coordinate system 
$O x y$ this problem is equivalent to a uniform stream with velocity $\nabla \Phi_{\infty}=\vec{U}_{\infty}=-\vec{U}_{B}$, where $\Phi_{\infty}(\mathbf{P})=u_{\infty} x+v_{\infty} y$ is the far-field asymptotic form of the velocity potential $\Phi(\mathbf{P})$ of the resulting flow at point $\mathbf{P}=(x, y)$. The potential $\Phi(\mathbf{P})$ is the solution of the following boundaryvalue problem $(\mathrm{BVP})$ :

$$
\begin{aligned}
\nabla^{2} \Phi=0, \quad \mathbf{P}=(x, y) \in \Omega, \\
\frac{\partial \Phi}{\partial n}=0, \quad \mathbf{P} \in \partial \Omega_{B}, \\
\Phi-\left(u_{\infty} x+v_{\infty} y\right) \rightarrow 0, \quad \text { as } x^{2}+y^{2} \rightarrow \infty,
\end{aligned}
$$

where $\Omega$ is the fluid domain outside $\partial \Omega_{B}$ and $\vec{n}$ denotes the unit normal vector on $\partial \Omega_{B}$ directed inwards with respect to the body. The above BVP has a unique solution up to an additive constant and, in order to fix a unique solution, we normally consider, for smooth bodies, zero circulation $\Gamma(C)=\int_{C} \nabla \Phi \cdot d \mathbf{c}$ of the velocity field $\nabla \Phi$ over any circuit $C$ surrounding the body. The difference between potential flows around a smooth body and a hydrofoil is that, in order for the flow around the hydrofoil to have a physical meaning, the circulation has to be nonzero and appropriately adjusted until the flow leaves the trailing edge smoothly. More specifically, on the basis of Kelvin's theorem, Prandtl concluded that if an airfoil, which started its motion from rest in an ideal fluid, is later found to possess non-zero circulation $\Gamma$, then the component of the boundary of the fluid which coincided with the airfoil initially, must coincide at a later time with the union of the airfoil surface and a surface, the so-called wake, embedded in the fluid which has circulation $-\Gamma$; see, e.g., Meyer (1971) [36]. In contrast to the 3D case (see, e.g., Bassanini et al (1999) [37]), the location and shape of the wake in the 2D case can be taken, without loss of generality, to be a straight line emanating from the trailing edge and extending to infinity. This line is a force-free boundary along which the normal fluid velocity and the pressure should exhibit no jump. More accurately, we can write:

$$
\begin{aligned}
\frac{\partial \Phi^{+}}{\partial n} & =\frac{\partial \Phi^{-}}{\partial n}, \quad \mathbf{P} \in \partial \Omega_{w}: \text { kinematic boundary condition, } \\
p^{+} & =p^{-}, \quad \mathbf{P} \in \partial \Omega_{w}: \text { dynamic boundary condition }
\end{aligned}
$$

where, $\Phi^{ \pm} / p^{ \pm}$denote the velocity potential / pressure on the upper, $\partial \Omega_{w^{+}}$, and lower, $\partial \Omega_{w^{-}}$, face of the wake boundary, respectively.

Let $\Omega$ be the fluid computational domain bounded by $\partial \Omega=\partial \Omega_{B} \cup \partial \Omega_{w^{+}} \cup \partial \Omega_{\infty} \cup \partial \Omega_{w^{-}}$with the radius of the fictitious circular boundary $\partial \Omega_{\infty}$ tending to infinity and the angle $\theta$ between the fictitious boundaries $\partial \Omega_{w^{+}}$and $\partial \Omega_{w^{-}}$tending to zero as $r \rightarrow \infty$. Applying in $\Omega$ Green's second identity between the potential $\Phi(\mathbf{P}), \mathbf{P} \in \Omega$, and the fundamental solution, $G(\mathbf{P}, \mathbf{Q})=$ $(1 / 2 \pi) \ln \|\mathbf{P}-\mathbf{Q}\|$, of the 2D Laplace equation (1), we can reformulate the BVP (1)-(3) as a $2^{n d}$-kind Fredholm integral equation on the hydrofoil boundary $\partial \Omega_{B}$, taking into account the wake-sheet conditions (4) and (5):

$$
\frac{\Phi(\mathbf{P})}{2}+\int_{\partial \Omega_{B}} \Phi(\mathbf{Q}) \frac{\partial G(\mathbf{P}, \mathbf{Q})}{\partial n_{Q}} d s_{Q}-\mu_{w} \int_{\partial \Omega_{w}} \frac{\partial G(\mathbf{P}, \mathbf{Q})}{\partial n_{Q}} d s_{Q}=\Phi_{\infty}(\mathbf{P}), \quad \mathbf{P} \in \partial \Omega_{B} \backslash \mathbf{P}_{T E},
$$

where subscript $Q$ implies an operator with respect to point $\mathbf{Q}, \mathbf{P}_{T E}=\left(x_{e}, y_{e}\right)$ denotes the trailing edge of the hydrofoil and

$$
\mu_{w}=\Phi^{+}(\mathbf{P})-\Phi^{-}(\mathbf{P})=\text { constant }, \quad \mathbf{P} \in \partial \Omega_{w} .
$$

Equation (7) is a form of the Kutta condition, the so-called Morino-Kutta condition (see Morino \& Kuo (1974) [22], stating that the fluid velocity should be bounded in $\Omega$ and in particular near the trailing edge, enabling to fix the proper value of circulation $\Gamma$, thus securing a unique solution 


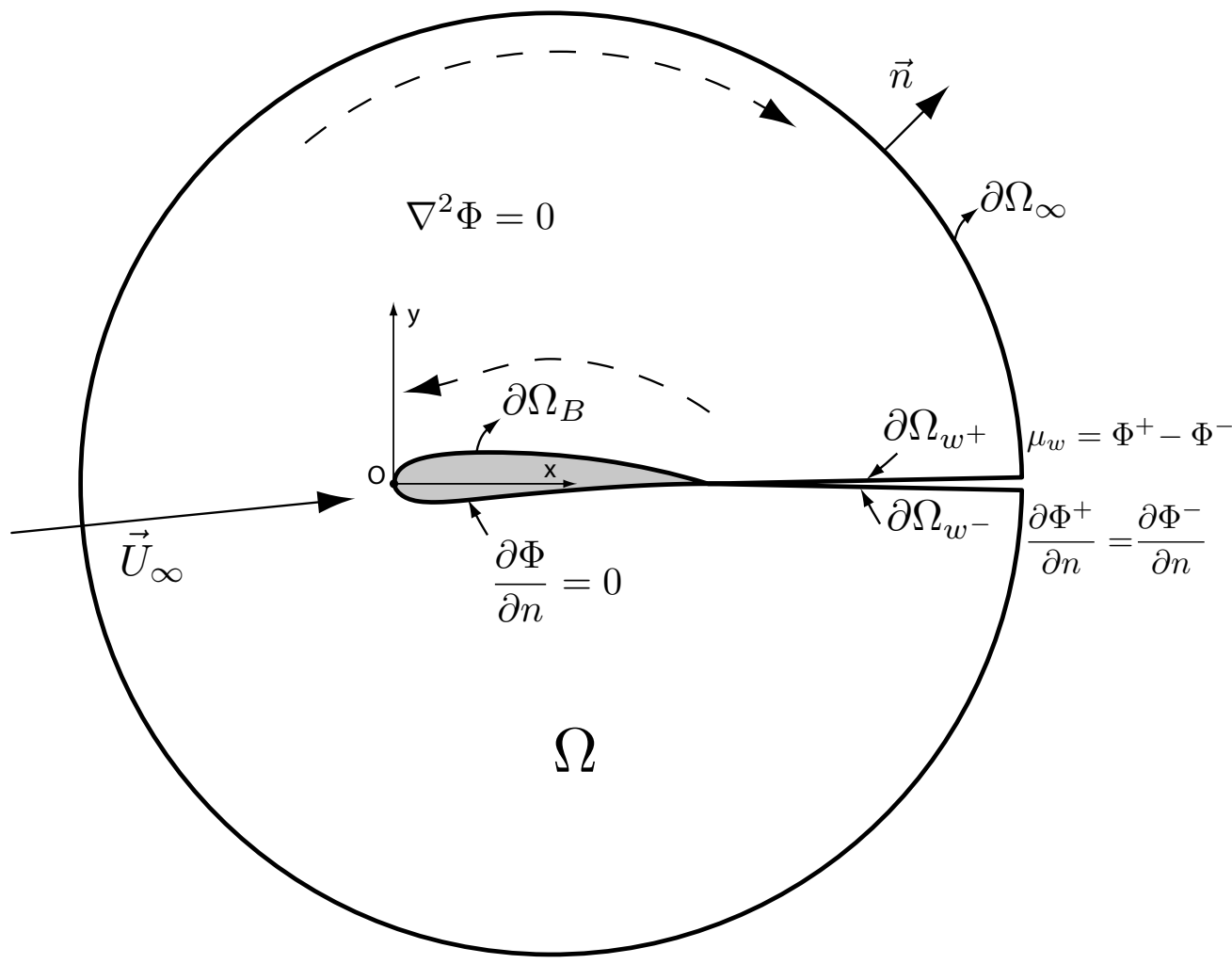

Figure 1: A hydrofoil in a incoming uniform stream with velocity $\vec{U}_{\infty}$ along with its wake boundary $\partial \Omega_{w}$. Dashed lines denote the assumed boundary orientation.

of the BVP (1)-(3). Furthermore, due to the simple shape of the wake sheet, the integral term on the wake $\partial \Omega_{w}$, appearing in equation (6), can be easily calculated resulting in:

$$
\int_{\partial \Omega_{w}} \frac{\partial G(\mathbf{P}, \mathbf{Q})}{\partial n_{Q}} d s_{Q}=\int_{x_{e}}^{\infty} \frac{\partial G\left(\mathbf{P},\left(x_{Q}, y_{Q}\right)\right)}{\partial n_{Q}} d x_{Q}=\frac{1}{2 \pi} \arctan \left(\frac{y_{P}-y_{e}}{x_{P}-x_{e}}\right),
$$

revealing that the influence of the wake to the fluid flow is equivalent to a point vortex located at the trailing edge $\mathbf{P}_{T E}=\left(x_{e}, y_{e}\right)$. Alternatively, the flow around the hydrofoil can be formulated via the perturbation potential $\phi(\mathbf{P})$, defined as:

$$
\phi(\mathbf{P})=\Phi(\mathbf{P})-\Phi_{\infty}(\mathbf{P}),
$$

which leads to the following integral equation:

$$
\begin{array}{r}
\frac{\phi(\mathbf{P})}{2}+\int_{\partial \Omega_{B}} \phi(\mathbf{Q}) \frac{\partial G(\mathbf{P}, \mathbf{Q})}{\partial n_{Q}} d s_{Q}-\mu_{w} \int_{\partial \Omega_{w}} \frac{\partial G(\mathbf{P}, \mathbf{Q})}{\partial n_{Q}} d s_{Q}= \\
-\int_{\partial \Omega_{B}}\left(\vec{U}_{\infty} \cdot \vec{n}(\mathbf{Q})\right) G(\mathbf{P}, \mathbf{Q}) d s_{Q}, \quad \mathbf{P} \in \partial \Omega_{B} \backslash \mathbf{P}_{T E} .
\end{array}
$$

The range of the variable point $\mathbf{P}$ in the above integral equation, as well as in Eq. 6, does not include the trailing-point $\mathbf{P}_{T E}$ in order to circumvent the issue that, due to the existence of the wake $\partial \Omega_{w}$ emanating from $\mathbf{P}_{T E}$, one cannot simply appeal to standard potential theory for boundaries $\partial \Omega_{B}$ with corners and replace the term $\Phi\left(\mathbf{P}_{T E}\right) / 2$ in the left-hand side of Equations 6 and 10 by $[1-(\hat{\beta} / 2 \pi)] \Phi\left(\mathbf{P}_{T E}\right), \hat{\beta}$ denoting the internal angle at the trailing edge. This choice controls the collocation method, adopted in $\S 2.1$, for solving the IGA-BEM discretization of (10), 
by restricting the first and last collocation points from collapsing on $\mathbf{P}_{T E}$. There exists, however, works in pertinent literature, e.g., Morino \& Bernardini (2001) [38], where $\mathbf{P}_{T E}$ is permitted to be a collocation point in the context of a an enhanced formulation that involves an additional integral equation resulting from taking the derivative of the potential $\Phi(\mathbf{P}), \mathbf{P} \in \Omega$, with respect to the normal of the wake and letting $\mathbf{P} \rightarrow \mathbf{P}_{T E}$.

After solving (6) or (10), we can calculate the hydrofoil's pressure coefficient $c_{p}$ using Bernoulli's equation:

$$
c_{p}:=\frac{p-p_{\infty}}{\frac{1}{2} \rho\left|\vec{U}_{\infty}\right|^{2}}=1-\frac{v_{t}^{2}}{\left|\vec{U}_{\infty}\right|^{2}},
$$

where $v_{t}$ is the tangential fluid velocity on the hydrofoil's $\partial \Omega_{B}$ given by $v_{t}=\partial \Phi / \partial s$ with $s$ denoting the arc length on $\partial \Omega_{B}$.

\subsection{A discrete IGA-BEM formulation}

The purpose of this subsection is to present a method that combines Boundary Element Method (BEM) with IsoGeometric Analysis (IGA) for solving numerically the boundary integral equations (6) and (10). IGA philosophy is equivalent to approximating the field quantities (dependent variables) of the boundary-value problem in question by the very same basis that is being used for representing (accurately) the geometry of the involved body-boundary. In our case the dependent variables are the total potential $\Phi(\mathbf{P}), \mathbf{P} \in \partial \Omega_{B}$ (see eq. (6)) or the perturbation potential $\phi(\mathbf{P}), \mathbf{P} \in \partial \Omega_{B}$ (see eq. (10)). For this purpose, we shall presume that the body boundary $\partial \Omega_{B}$ can be (accurately) represented as a closed parametric NURBS curve $\mathbf{r}(t), t \in[0,1]$, which is regular, i.e., the derivative vector is well defined and not vanishing, with the exception of the trailing edge: $\mathbf{r}(0)=\mathbf{r}(1)$, where the derivative vector is not defined. More specifically,

$$
\mathbf{r}(t)=(x(t), y(t)):=\sum_{i=0}^{n} \mathbf{d}_{i} M_{i, k}(t), \quad t \in I=\left[t_{k-1}, t_{n+1}\right]:=[0,1],
$$

where $\left\{M_{i, k}(t)\right\}_{i=0}^{n}$ is a rational $B$-spline basis of order $k$, defined over a knot sequence $\mathcal{J}=$ $\left\{t_{0}, t_{1}, \ldots, t_{n+k}\right\}$ and possessing non-negative weights $w_{i}, i=0, \ldots, n$, while $\mathbf{d}_{i}$ are the associated control points; see, e.g., Piegl \& Tiller [39]. Equation (10) can then be written in the following form:

$$
\frac{\phi(t)}{2}+\int_{I} \phi(\tau) K(t, \tau) d \tau-\frac{\mu_{w}}{2 \pi} \arctan \left(\frac{y(t)-y_{e}}{x(t)-x_{e}}\right)=g(t), \quad t \in(0,1),
$$

where, for the sake of notational simplicity, we define $\phi(t):=\phi(\mathbf{r}(t)), G(t, \tau)):=G(\mathbf{r}(t), \mathbf{r}(\tau))$ and $K(t, \tau)=\left(\partial G(t, \tau) / \partial n_{\tau}\right)\|\dot{\mathbf{r}}(\tau)\|, g(t)=-\int_{I}\left(\vec{U}_{\infty} \cdot \vec{n}(\tau)\right) G(t, \tau)\|\dot{\mathbf{r}}(\tau)\| d \tau$. The kernel $K(t, \tau)$ of the integral equation (13) is a function expressed as:

$$
K(t, \tau)=\frac{\dot{\mathbf{r}}(\tau) \times(\mathbf{r}(t)-\mathbf{r}(\tau))}{2 \pi\|\mathbf{r}(t)-\mathbf{r}(\tau)\|^{2}} \quad \text { if } \quad t \neq \tau,
$$

where here the symbol $\times$ denotes the so-called "2D exterior product" defined as: $\left(x_{1}, y_{1}\right) \times$ $\left(x_{2}, y_{2}\right):=x_{1} y_{2}-x_{2} y_{1}$. Assuming that $\mathbf{r}(\tau) \in C^{2}(I)$, a careful limiting process as $\tau \rightarrow t$ involving a $2^{\text {nd }}$-order Taylor expansion of $\mathbf{r}(\tau)$ around $\tau=t$, gives:

$$
K(t, t)=\frac{\dot{\mathbf{r}}(t) \times \ddot{\mathbf{r}}(t)}{2 \pi\|\dot{\mathbf{r}}(t)\|^{2}}, \quad \text { if } \quad t=\tau,
$$

revealing that in the neighborhood of regular points of a smooth (at least $C^{2}$ ) $\partial \Omega_{B}$, the kernel $K(t, \tau)$ is a regular function. 
Aiming to employ IGA for handling equation (13), we project, in a suitably defined manner, the perturbation potential $\phi(t)$ on the spline space $\mathcal{S}^{k}\left(\mathcal{J}^{(\ell)}\right), \mathcal{S}^{k}\left(\mathcal{J}^{(0)}\right):=\mathcal{S}^{k}(\mathcal{J})$, expressed in the form:

$$
\phi_{s}(t):=\mathcal{P}_{s}(\phi(t))=\sum_{i=0}^{n+\ell} \phi_{i} M_{i, k}^{(\ell)}(t), \quad t \in I, M_{i, k}^{(0)}(t):=M_{i, k}(t),
$$

where $\ell \in \mathbb{N}_{0}$ denotes the number of knots inserted in $I$. Recalling the fundamental property of knot insertion, we can say that $\left\{\mathcal{S}^{k}\left(\mathcal{J}^{(\ell)}\right), \ell \in \mathbb{N}_{0}\right\}$ constitutes a sequence of nested finite dimensionalspaces, i.e., $\mathcal{S}^{k}\left(\mathcal{J}^{(\ell)}\right) \subset \mathcal{S}^{k}\left(\mathcal{J}^{(\ell+1)}\right)$. Equation (13) can then be written as

$$
\frac{1}{2} \sum_{i=0}^{n+\ell} \phi_{i} M_{i, k}^{(\ell)}(t)+\int_{I} \sum_{i=0}^{n+\ell} \phi_{i} M_{i, k}^{(\ell)}(\tau) K(t, \tau) d \tau-\frac{\mu_{w}}{2 \pi} \arctan \left(\frac{y(t)-y_{e}}{x(t)-x_{e}}\right)=g(t), \quad t \in(0,1) .
$$

Several methods are available for defining the projection $\mathcal{P}_{s}$ (see eq.16) onto the finite-dimensional space $\mathcal{S}^{k}\left(\mathcal{J}^{(\ell)}\right)$ and discretizing equation (13), like Galerkin and collocation. In the present work, a collocation scheme is adopted, which consists in projecting on $\mathcal{S}^{k}\left(\mathcal{J}^{(\ell)}\right)$ through interpolation at a set of collocation points $t=t_{j}, j=0, \ldots, n+\ell$, which are chosen to be the Greville abscissas associated with the knot vector $\mathcal{J}^{(\ell)}$. This leads to the following linear system for the unknown coefficients $\phi_{i}, i=0, \ldots, n+\ell$ :

$$
\frac{1}{2} \sum_{i=0}^{n+\ell} \phi_{i} M_{i, k}^{(\ell)}\left(t_{j}\right)+\sum_{i=0}^{n+\ell} \phi_{i} q_{i}\left(t_{j}\right)-\frac{\left(\phi_{n+\ell}-\phi_{0}\right)}{2 \pi} \arctan \left(\frac{y\left(t_{j}\right)-y_{e}}{x\left(t_{j}\right)-x_{e}}\right)=g\left(t_{j}\right), \quad j=0, \ldots n+\ell,
$$

where $q_{i}\left(t_{j}\right)=\int_{I} M_{i, k}^{(\ell)}(\tau) K\left(t_{j}, \tau\right) d \tau$ while, taking into account (7), the unknown $\mu_{w}$ is expressed through the difference $\phi_{n+\ell}-\phi_{0}$. Since $t \in(0,1)$, one must consider shifting the values of the first and the last Greville abscissa by a small value $\epsilon>0$. This shifting process gives rise to the need of proper handling of the numerical evaluation of the integral terms $q_{0}(1-\epsilon)$ and $q_{n+\ell}(\epsilon)$ appearing in (18). Indeed, by using the fact that $\mathbf{r}(0)=\mathbf{r}(1)$ and incorporating $1^{\text {st }}$-order Taylor expansion of $\mathbf{r}(\epsilon), \dot{\mathbf{r}}(\epsilon)$ and $\mathbf{r}(1-\epsilon), \dot{\mathbf{r}}(1-\epsilon)$ around 0 and 1, respectively, equation (14) leads to the following asymptotic estimates for the kernel:

$$
K(1-\epsilon, \epsilon)=O\left(\frac{1}{\epsilon}\right), \quad K(\epsilon, 1-\epsilon)=O\left(\frac{1}{\epsilon}\right), \quad K(1-\epsilon, 0)=O\left(\frac{1}{\epsilon}\right), \quad K(\epsilon, 1)=O\left(\frac{1}{\epsilon}\right) .
$$

Thus, since $M_{0, k}^{(\ell)}(\epsilon)=O(1), M_{n+\ell, k}^{(\ell)}(1-\epsilon)=O(1)$, we conclude that the integrands of $q_{0}(1-\epsilon)$ and $q_{n+\ell}(\epsilon)$ are of order of $1 / \epsilon$ in a neighborhood of 0 and 1, respectively. Actually, as one can see in the attached media file ${ }^{1}$, this behavior is restricted in an $\epsilon$ neighborhood of 0 and 1 and the graph of the integrands have a bell-like shape with a height of $O(1 / \epsilon)$ and width of $O(\epsilon)$. These integrals can be handled by an adaptive numerical integration scheme provided that precautions are taken in order that $\epsilon$ does not fall under a threshold value for which the integrals cannot be numerically computed.

The solution of the linear system (18) provides the values $\phi_{i}, i=0, \ldots, n+\ell$, which can be used to easily calculate the tangential velocity on the hydrofoil by using the derivatives of the rational B-spline basis functions $M_{i, k}^{(\ell)}(t)$, without resorting to the finite difference scheme, as it is the the practice in low-order panel methods:

$$
v_{t}=\vec{U}_{\infty} \cdot \vec{t}+\frac{\partial \phi}{\partial s}=\vec{U}_{\infty} \cdot \vec{t}+\frac{1}{\|\dot{\mathbf{r}}(t)\|} \sum_{i=0}^{n+\ell} \phi_{i} \frac{d M_{i, k}^{(\ell)}(t)}{d t},
$$

where $\vec{t}$ denotes the unit-tangent vector of $\mathbf{r}(t)$.

\footnotetext{
${ }^{1}$ See online version of the document.
} 


\section{Numerical results \& optimization examples}

As a first attempt to test the accuracy and effectiveness of the IGA-BEM method, presented in $§ 3.3$, we present numerical results for the flow around the standard NACA-4412 profile. This hydrofoil is represented as a cubic B-spline curve with 47 knots and 43 control points.

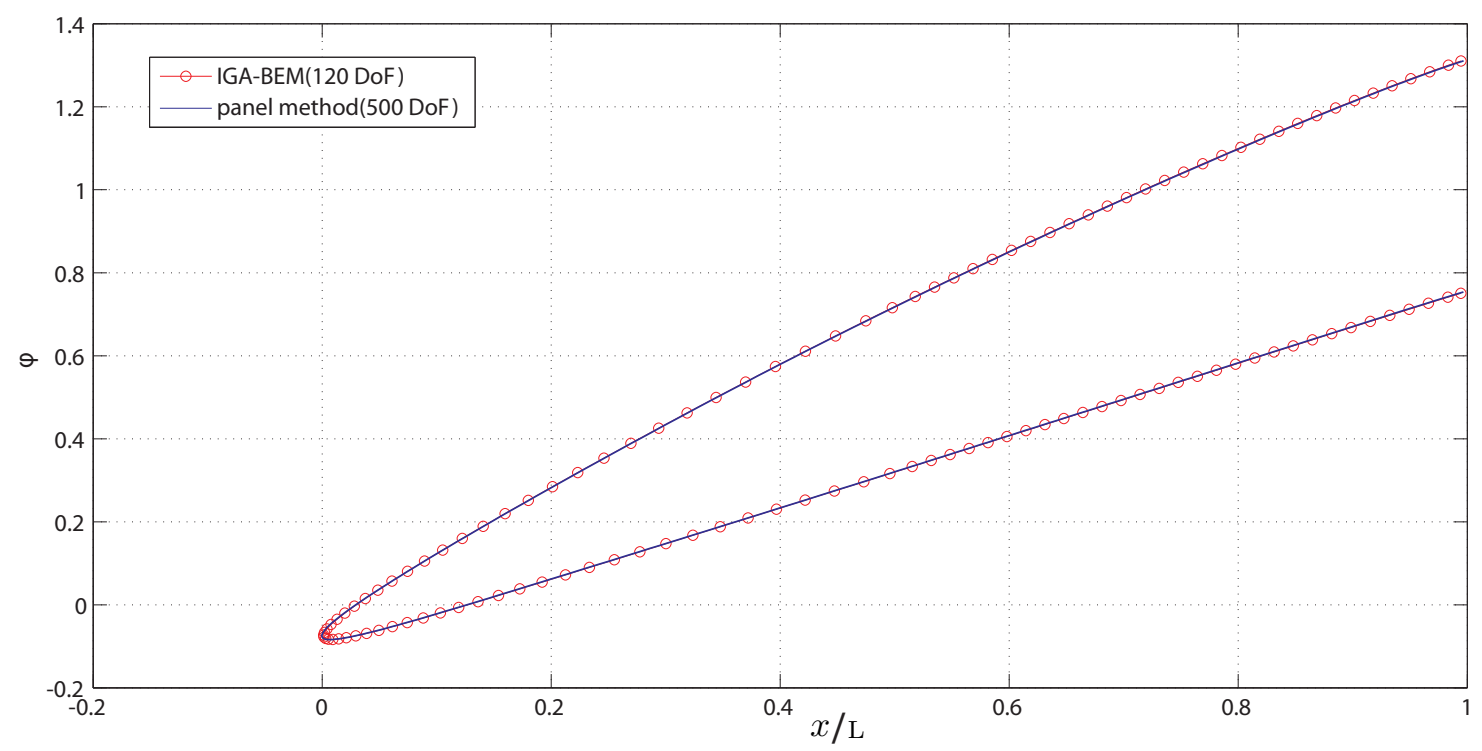

Figure 2: Potential distribution around a NACA-4412 profile at 5 deg. angle of attack and comparison with low-order panel method (L denotes the chord length).

In Figure 2, the total potential $\Phi$ on the hydrofoil is presented for an incident flow at an angle of attack of 5 deg. Numerical results of the present IGA method are compared with corresponding results of low-order panel method (Moran 1984) [40] using a large number of elements $(\mathrm{N}=500)$. The agreement of IGA solution, using considerably less degrees of freedom $(n=120)$, is very satisfactory. We recall that in IGA method degrees of freedom (DoF) is the number of the control coefficients used in the NURBS approximation of the potential $\Phi$; see eq. (16).

In Figure 3, we compare the pressure coefficient distribution around a NACA-4412 profile at $7 \mathrm{deg}$. angle of attack, with corresponding results with a low-order panel method which adopts either the direct (Morino) formulation (see [40]), as in the present work, or the classical indirect formulation of Hess \& Smith [20]. Again, considering the low-order panel solution with a large number of elements $(\mathrm{N}=500)$, we can see the good performance of IGA method, where it is needed considerably less DoF $(\mathrm{n}=120)$ for the solution to converge.

In Figure 4, we compare the overall performance of IGA-BEM compared to low-order BEM. This is accomplished by plotting, for both methods, the error of the developed circulation of the flow $\mu_{w}$ around the hydrofoil (we recall that $\mu_{w}=\phi_{n+\ell}-\phi_{0}$; see eq. (7)) as a function of DoF. Since the exact circulation around the hydrofoil is not known, the error calculated is based on a reference solution, $\mu_{w r}$, which is computed using the IGA-BEM method for a large number of DoF $(\simeq 5000)$. In IGA-BEM refinement is performed by knot insertion (h-refinement). It can be observed that IGA-BEM performs better compared to low-order panel method (e.g. for a relative accuracy of 0.001 the IGA approach requires around $120 \mathrm{DoFs}$, while for the low-order panel method this rises to 1000 DoFs; see also Figures 2 and 3). For this level of accuracy the computational time is 17 and 24 seconds for the IGA and panel method respectively, when executed on a standard desktop PC. The rate of convergence exhibited by the IGA method is superior to that of the panel one but not analogous to the corresponding results concerning potential flows without circulation (see [29]) and application of the present IGA-BEM method in elasticity problems (see, e.g.,[11] and [30]). This behavior may be attributed to the trailing edge, where the body boundary ceases to be smooth and the wake sheets emanate. 


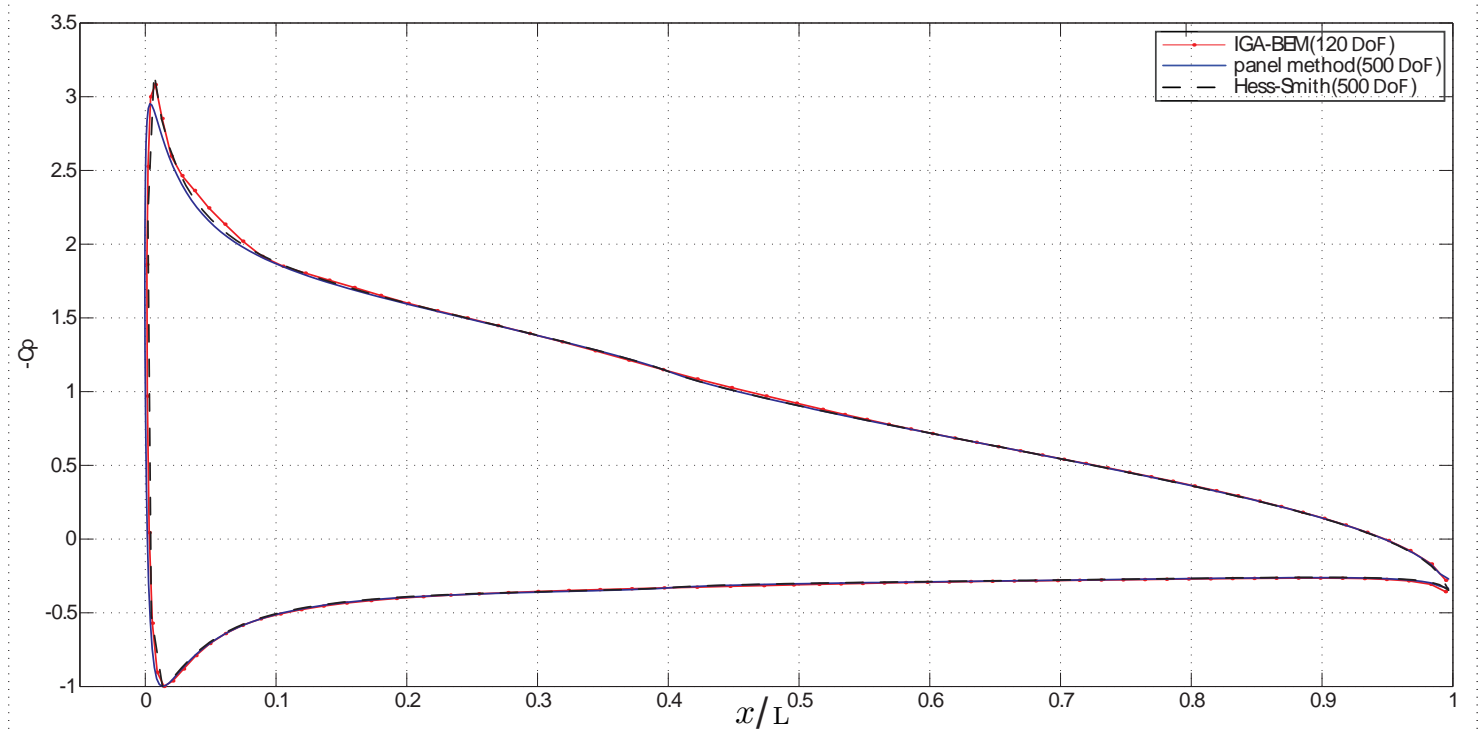

Figure 3: Pressure distribution around a NACA-4412 profile at 7 deg. angle of attack and comparison with low-order panel methods

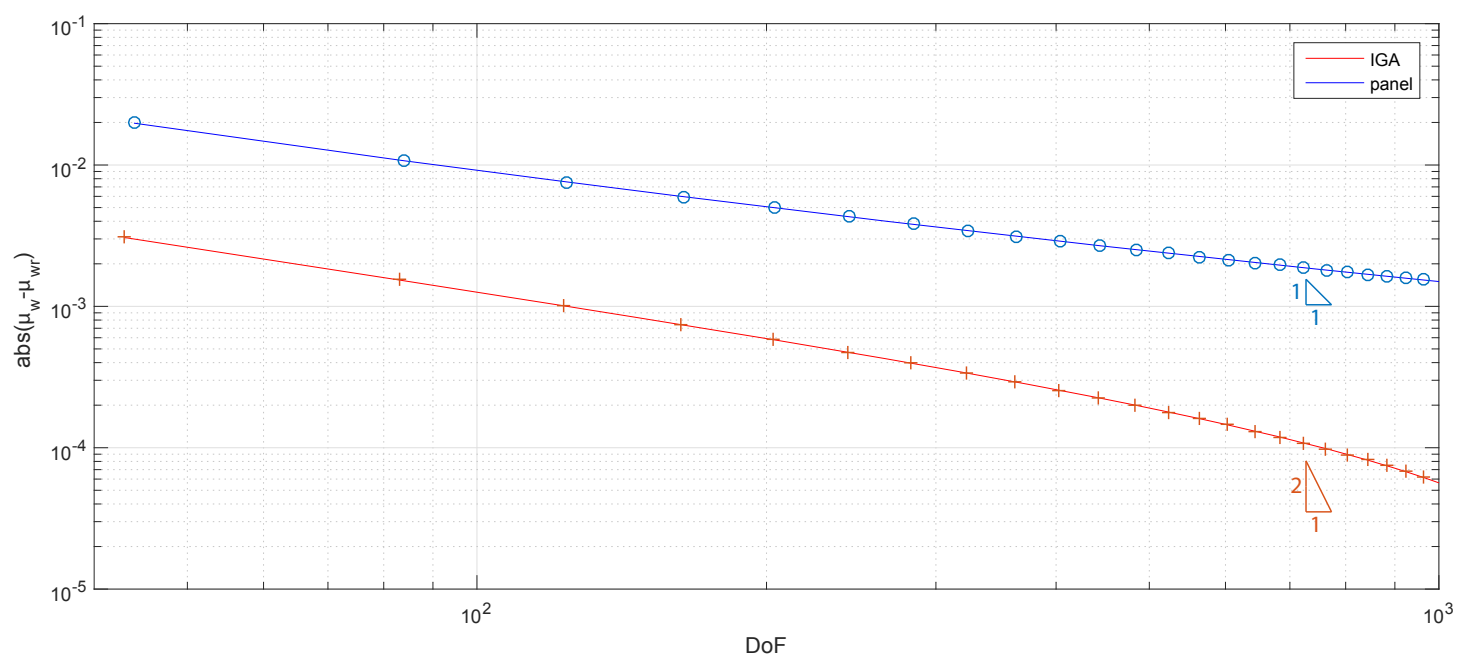

Figure 4: Error of the circulation $\mu_{w}$ around a NACA-4412 hydrofoil wrt DoF 


\subsection{Optimization environment}

Simulation-based optimization is of growing importance in engineering, since it allows to improve a component's or system's performance for moderate cost, in comparison with generally costly physical experiments. Moreover, the optimization is conducted in a rigorous algorithmic framework that enhances the experience and intuition of designers. A major difficulty to apply an automated shape optimization for complex engineering systems is the development of a fully automated design loop. Specifically, for each optimizer-generated set of parameters, a valid geometric model has to be constructed, allowing the generation of the computational domain used by the solver to provide the physical response and the performance analysis which needs to be returned back to the optimizer. All these steps should be fully automated, without user intervention, in order to feed the optimization algorithm and finalize the design loop. In this context, the IGA paradigm offers a significant improvement over the classical panel-based methods, since it relies on a direct relationship between the design parameters and the solver, without any geometrical intermediate meshes.

A second obstacle arises from the simulation process: for complex test-cases, CFD simulations are expensive, in terms of computational time. Moreover, the numerical solutions obtained can be polluted by errors arising from the discretization and iterative methods, yielding noisy performance evaluations. Sometimes, this may lead the optimizer to spurious local optima or even yield the failure of the optimization procedure. Here again, the isogeometric context may be helpful because it allows to avoid geometrical approximations, which reduces the error level, and permits to construct high-order solutions yielding a better computational efficiency.

In our case, the optimization loop comprises three components: the optimization algorithm, the IGA solver presented in $\S 2$ and the hydrofoil parametric model which will be presented in the following subsection. Hydrofoil shape optimization examples using the developed framework are presented in $\S 3.3$. Both the optimizer and IGA solver are implemented in MATLAB while the hydrofoil parametric model is implemented in Rhinoceros ${ }^{\circledR} 3 \mathrm{D}$ modeling package.

The selected optimization algorithm belongs to the category of evolutionary ones, as experimentation with gradient and hessian-based algorithms has indicated the existence of multiple local minima that makes their usage problematic. Specifically, our optimizer uses the multi-objective optimization method gamultiobj which employs a controlled elitist genetic algorithm (a variant of NSGA-II [41]). An elitist Genetic Algorithm (GA) always favors individuals with better fitness value (rank). A controlled elitist GA also favors individuals that can help increase the diversity of the population even if they have a lower fitness value. It is important to maintain the diversity of population for convergence to an optimal Pareto front.

\subsection{Parametric Model for a Hydrofoil}

The parametric model for a general hydrofoil has been materialized within Rhinoceros ${ }^{\circledR} 3 \mathrm{D}$ modeling software package with the aid of its VBscript-based programming language, Rhinoscript. Our model generates a closed cubic B-Spline curve that represents a hydrofoil, using a set of 8 parameters; see Table 1. The geometrical definition of these parameters is depicted in Figure 5. All parameters, with the exception of chord's length (L), are defined using appropriate nondimensional ratios so that their values always lie in $[0,1]$. This approach eliminates the need of implementing complex interdependent constraints while guaranteeing the robustness of the procedure which is of significant importance in an optimization procedure. The dimensionalized range of the parameters is appearing in the "actual range" column of Table 1.

The parametric model's construction initiates with the definition of four simple Bézier curves that are employed in the generation of the final cubic B-Spline curve. The upper part of the hydrofoil comprises two parts: a simple cubic Bézier curve and a simple quadratic one for the fore and aft part respectively. The first control point of the cubic curve is positioned at $(0,0)$ while the last one is at (x_z_max, z_max). The first interior point lie on the line segment defined by $(0,0)$ and $\left(0, z_{-} \max \right)$ while the second one on the segment defined by $\left(0, z_{-} \max \right)$ and (x_z_max, $\left.z_{-} \max \right)$. The exact position of these points is controlled by the tip form factor parameter. The second part is a quadratic simple Bézier adjacent to the first part and ending on the trailing edge, i.e., 
Table 1: Parameters' definition

\begin{tabular}{|c|c|c|c|c|}
\hline Nr. & Name & description & symbol & actual range \\
\hline 1 & Length & Length of hydrofoil's chord & $\mathrm{L}$ & free \\
\hline 2 & Max width & $\begin{array}{l}\text { Maximum width of suction } \\
\text { side wrt chord }\end{array}$ & $\max \_z$ & $\left.\frac{L}{500}, \frac{L}{5}\right]$ \\
\hline 3 & Camber width & $\begin{array}{l}\text { Camber maximum width wrt } \\
\text { chord }\end{array}$ & $\max _{-} \mathrm{c}$ & {$\left[0,0.91 \max \_z\right]$} \\
\hline 4 & $\begin{array}{l}\text { Max-width posi- } \\
\text { tion }\end{array}$ & $\begin{array}{l}\text { Longitudinal position of suc- } \\
\text { tion side's max width }\end{array}$ & $\mathrm{x} \_z \_\max$ & {$\left[\frac{\mathrm{L}}{5}, \frac{7 \mathrm{~L}}{10}\right]$} \\
\hline 5 & $\begin{array}{l}\text { Max-camber- } \\
\text { width position }\end{array}$ & $\begin{array}{l}\text { Longitudinal position of cam- } \\
\text { ber's max width }\end{array}$ & $\mathrm{x}_{-} \mathrm{c} \max$ & {$\left[0, \frac{3 L}{10}\right]+\frac{7 x_{-} z_{-} \max }{10}$} \\
\hline 6 & $\begin{array}{l}\text { Suction-side an- } \\
\text { gle }\end{array}$ & $\begin{array}{l}\text { Suction's side angle at trail- } \\
\text { ing edge wrt chord }\end{array}$ & $a \_b$ & $\arctan \left(\frac{\text { z_max }}{\text { L-x_z_max }}\right), 89$ \\
\hline 7 & Camber angle & $\begin{array}{l}\text { Camber angle at trailing edge } \\
\text { wrt chord }\end{array}$ & a_b_p & {$\left[0, \mathrm{a} \_\mathrm{b}\right]$} \\
\hline 8 & Tip & Leading edge form factor & tip & {$[0.1,0.9]$} \\
\hline
\end{tabular}

at $(\mathrm{L}, 0)$. Its interior control point is positioned at $\left(\frac{\mathrm{L}-z_{-} \max }{\tan \left(\mathrm{a}_{-}\right)}, \mathrm{z}_{-} \max \right)$ which ensures first order geometric continuity at (x_z_max, z_max).

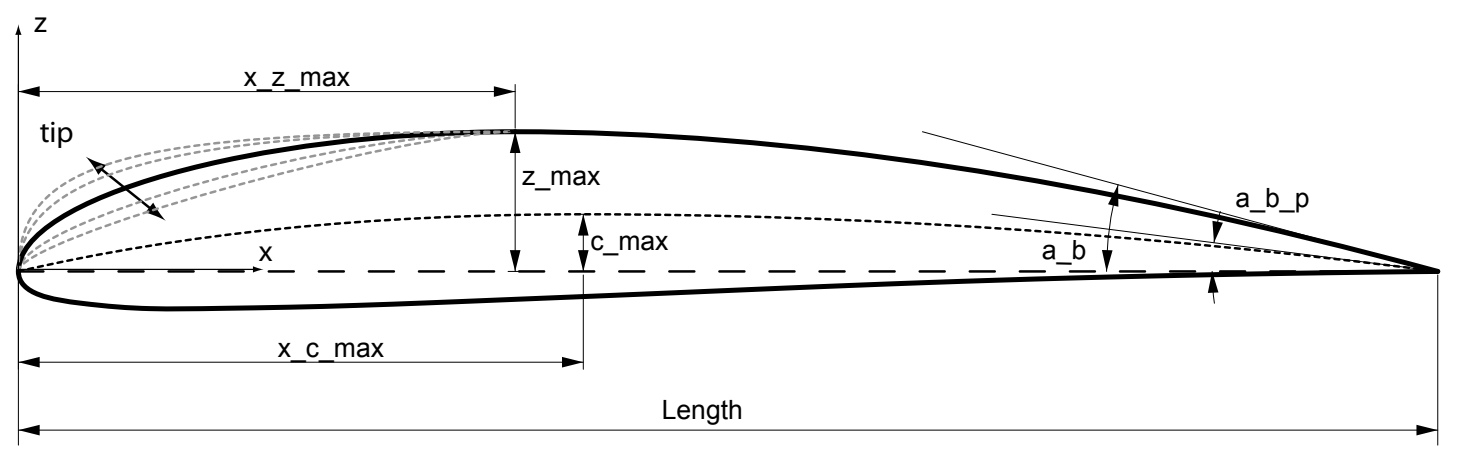

Figure 5: Parametric model of a hydrofoil and its defining parameters

The camber curve is constructed in a similar way using two quadratic simple Bézier curves. The aft part construction is equivalent to the aft part of the hydrofoil's suction side with $x_{z} z$ max , $z_{\_} \max$ and $a_{-} b$ being replaced by $x_{-} c_{\_} \max , c_{-} \max$ and $a_{-} b_{-} p$. For the fore part, an angular parameter is introduced, which corresponds to the tangent of the camber curve at the leading edge and uniquely defines the position of the interior control point. However, in our optimization examples this parameter has been kept fixed and is not included in the list of Table 1.

Finally, the pressure side is constructed by mirroring the upper part about the camber curve. The resulting three ${ }^{2}$ curve pieces can be exactly represented with a single cubic B-Spline curve, if quadratics are degree elevated and multiple knot insertion is performed at their endpoints. Alternatively, if knot multiplicity is to be avoided, the hydrofoil can be approximated by a single B-Spline curve using Rhinoscript's FitCurve ${ }^{3}$ function.

The developed parametric model is sufficiently flexible for our shape optimization purposes as it can easily generate specific hydrofoils, as the NACA-4412 depicted in Figure 6, or largely varying general hydrofoils; see shape instances in Figure 7. Figure 7(A) depicts a hydrofoil where

\footnotetext{
${ }^{2}$ Two for the suction side and one for the pressure side.

${ }^{3}$ FitCurve creates a B-Spline curve of specified degree that matches the input curve(s) within a specified tolerance value.
} 
the longitudinal positions of camber's and suction side's max-width have moved towards the trailing edge. Figures $7(\mathrm{~B}, \mathrm{D}, \mathrm{F}, \mathrm{H})$ depict mainly variations in the values of $\mathrm{z}$ max and $\mathrm{c}$ max, while Figures $7(\mathrm{~A}, \mathrm{E}, \mathrm{G})$ depict mainly variations in the relative positions of $\mathrm{x}_{-} z_{\_} \max$ and $\mathrm{x}_{-} \mathrm{c} \_$max. Finally, Figure $7(\mathrm{C})$ depicts a symmetric hydrofoil with c_max equaling 0.

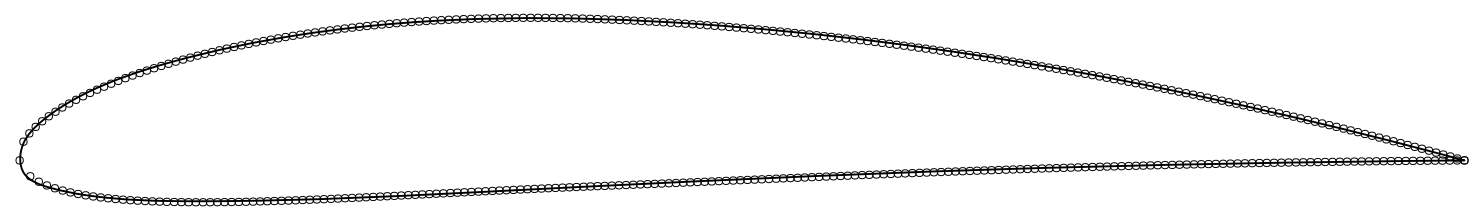

Figure 6: Approximation of a NACA-4412 profile using the parametric model. Max. deviation 0.1\% of chord length

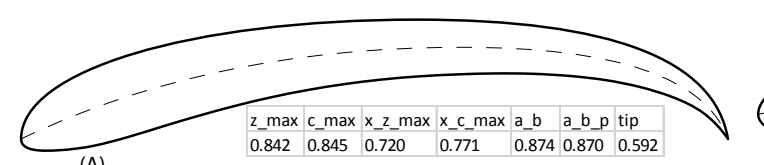

(A) $\begin{array}{lllllll}0.842 & 0.845 & 0.720 & 0.771 & 0.874 & 0.870 & 0.592\end{array}$
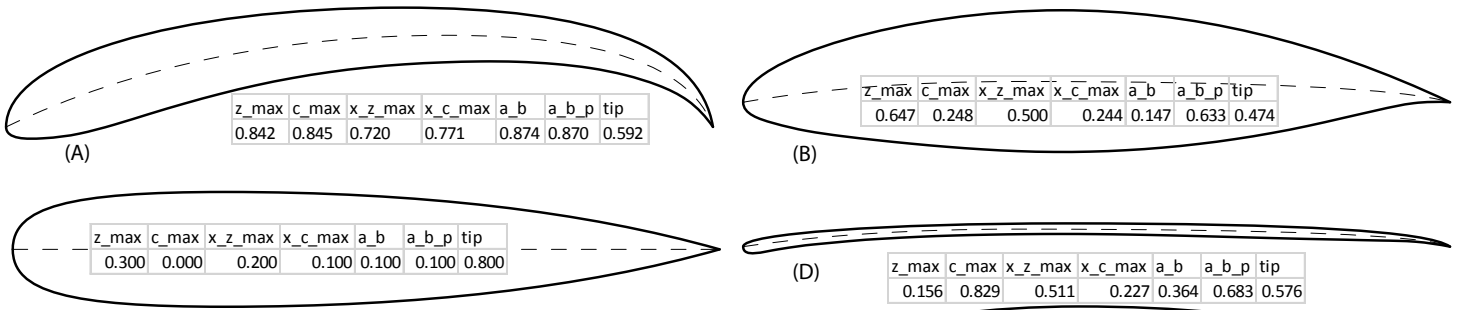

(C)

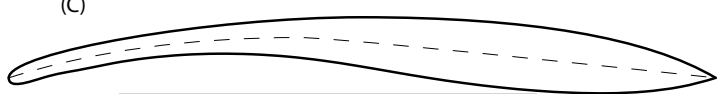

(E) z_max c_max x_z_max x_c_max a_b a_b_p tip $\begin{array}{llllllll}0.423 & 0.732 & 0.597 & 0.203 & 0.254 & 0.173 & 0.406\end{array}$
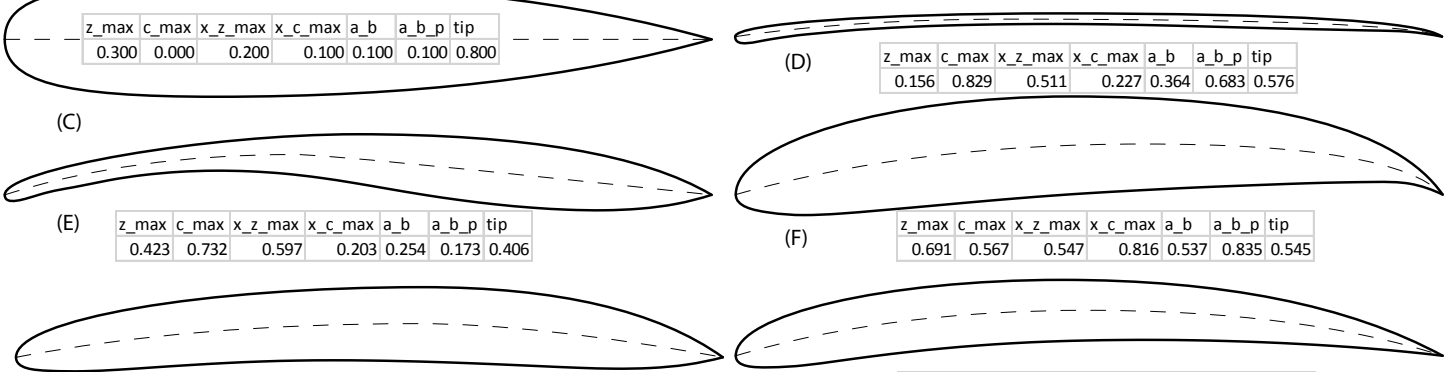

(G) z_max c_max x_z_max x_c_max a_b a_b_p tip $\begin{array}{llllllllll}0.491 & 0.528 & 0.751 & 0.553 & 0.286 & 0.266 & 0.552\end{array}$

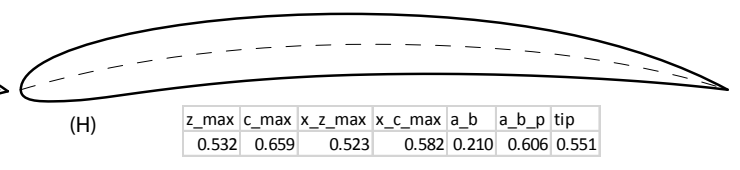

Figure 7: Various instances of the hydrofoil parametric model

\subsection{Shape optimization examples}

The optimization environment has been tested for optimizing the shape of a hydrofoil with respect to the following two criteria: a) maximum lift coefficient and b) minimum deviation from a reference area. The IGA-BEM solver, described in $\S 2.1$, is used to produce an average lift coefficient calculated for three angles of attack, namely 1, 3 and 5 degrees. The reference area in the second optimization criterion is set to be the one of the NACA-4412 profile. In the course of the optimization process, the parameter Length (L) of the hydrofoil parametric model is assumed to be fixed and is regularized to the value of one, while all remaining parameters, defined in Table 1, are considered to be free and contribute to the shape evolution of the hydrofoil.

As a first test, we consider that the values of the free parameters range between \pm 0.05 around those corresponding to the approximation of NACA-4412 by the parametric model (see Figure 6 ). Specifically, the min-max allowed values of the free parameters are given in Table 2. The multiobjective optimization method gamultiobj, offered by MATLAB, is employed to calculate the Pareto front of the above optimization problem, which is depicted in Figure 8. The Pareto front is defined to be the set of design points that are Pareto efficient, i.e., it is impossible to make any one of the objective function values better off without making the other (or more generally, at least one of the others) worse off. Obviously, the optimum shapes lie on the Pareto front; see green line depicted in Figure 8. If, for example, we choose to accept a $0.52 \%$ deviation of the target area, the optimum solution would be the hydrofoil instance whose parameter values are depicted in Table 2 . Table 2 includes both the non-dimensional and the corresponding dimensional (in parentheses) 
Table 2: NACA-4412 optimization parameters

\begin{tabular}{l|c|c|c|c}
\hline Nr. & Parameter & value for NACA4412 & range of variation & optimized \\
\hline 1 & L & $1(1)$ & fixed & 1 \\
\hline 2 & max_z & $0.487(0.0984)$ & {$[0.437,0.537]([0.0882,0.1083])$} & 0.459 \\
\hline 3 & max_c & $0.450(0.0403)$ & {$[0.400,0.500]([0.0358,0.0448])$} & 0.423 \\
\hline 4 & x_z_max & $0.300(0.3500)$ & {$[0.250,0.350]([0.3250,0.3750])$} & 0.259 \\
\hline 5 & x_c_max & $0.610(0.4280)$ & {$[0.560,0.660]([0.4130,0.4430])$} & 0.588 \\
\hline 6 & a_b & $0.070(0.2647)$ & {$[0.020,0.120]([0.1954,0.3340])$} & 0.110 \\
\hline 7 & a_b_p & $0.445(0.1275)$ & {$[0.395,0.495]([0.1151,0.1399])$} & 0.492 \\
\hline 8 & tip & $0.530(0.5240)$ & {$[0.480,0.580]([0.4840,0.5640])$} & 0.511
\end{tabular}

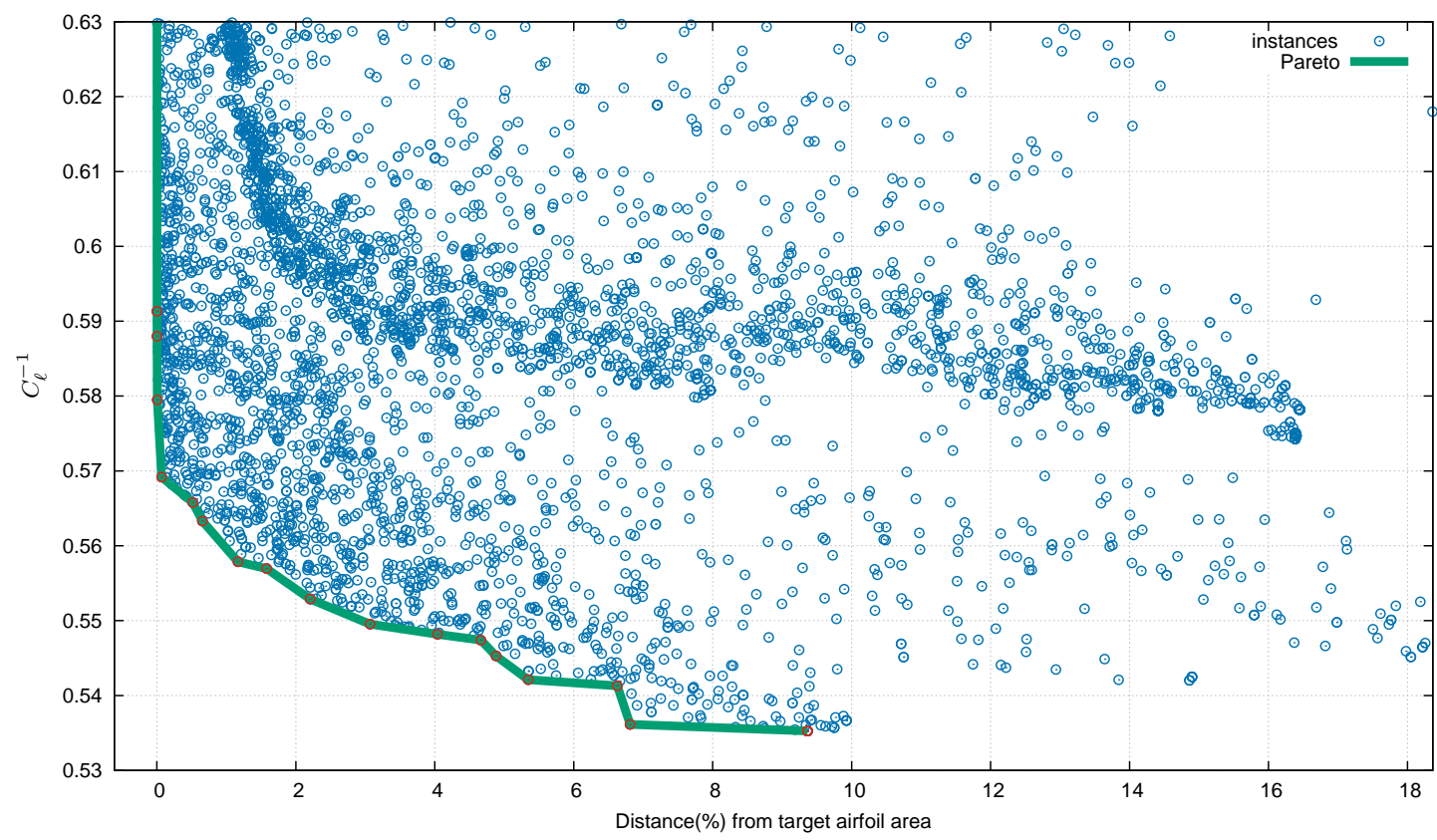

Figure 8: Pareto front for the inverse of the average lift coefficient, $C_{\ell}^{-1}$, and the area-deviation criteria. Average lift coefficient computed for 1,3 and 5 degrees of angle of attack. Parameters ranging \pm 0.05 around those corresponding to NACA-4412.

values of the parameters. The resulting "optimum" lift coefficient value is 1.768 , i.e., $\sim 3 \%$ better than that of NACA-4412. Finally, Figure 9, depicts the "optimum" instance of the hydrofoil along with an approximation of NACA-4412.

As a second test, we consider that the range of variation of the free parameters is not restricted and is the whole of the interval $[0,1]$. The resulting Pareto front is depicted in Figure 10. Finally, Figure 11 depicts six instances of the hydrofoil parametric model lying on the Pareto front. The hydrofoil instances in Figure 11 correspond to the points marked with the circled numbers in Figure 10. This second example leads to shapes that are prone to flow separation even for small Reynold's numbers. Hence, the potential flow theory should be carefully applied in this case and we may need to consider a different approach, e.g., boundary layer corrections or viscous flows, if we are to perform shape optimizations in large feasible spaces.

\section{Conclusions and future work}

In the present work, an optimization procedure has been developed and used for the shape optimization of hydrofoils. The optimization environment is based on a geometric parametric 


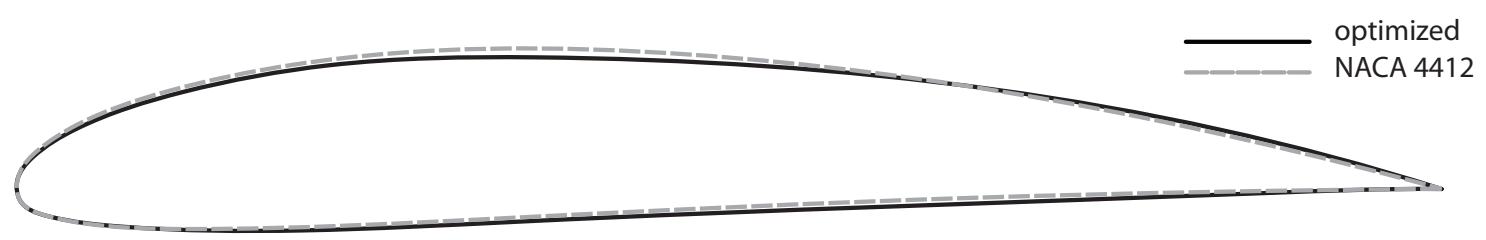

Figure 9: NACA-4412 along with an optimized instance on the Pareto front of Figure 8.

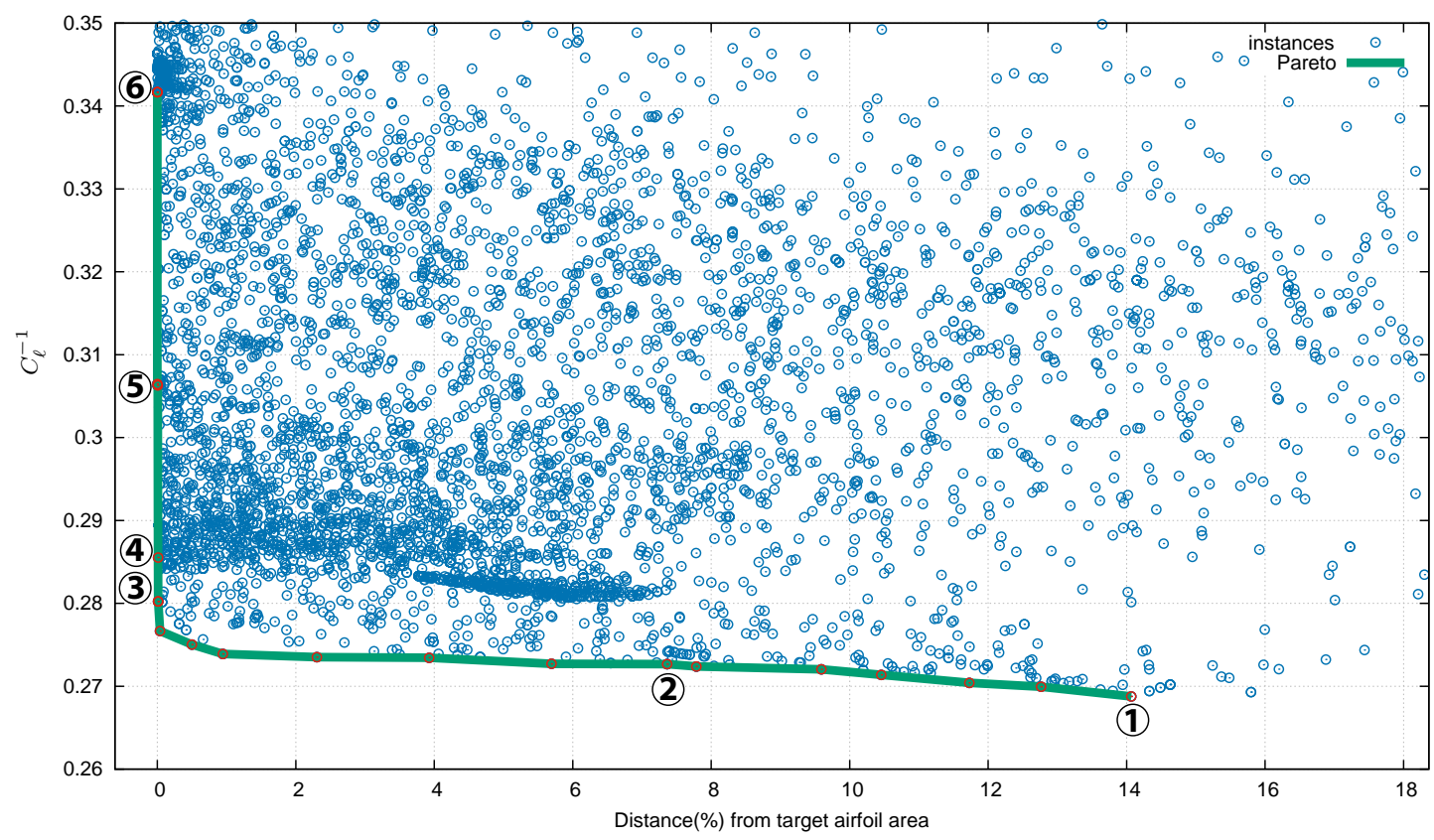

Figure 10: Pareto front for the inverse of the average lift coefficient and the area-deviation criteria. Average lift coefficient computed for 1,3 and 5 degrees of angle of attack.
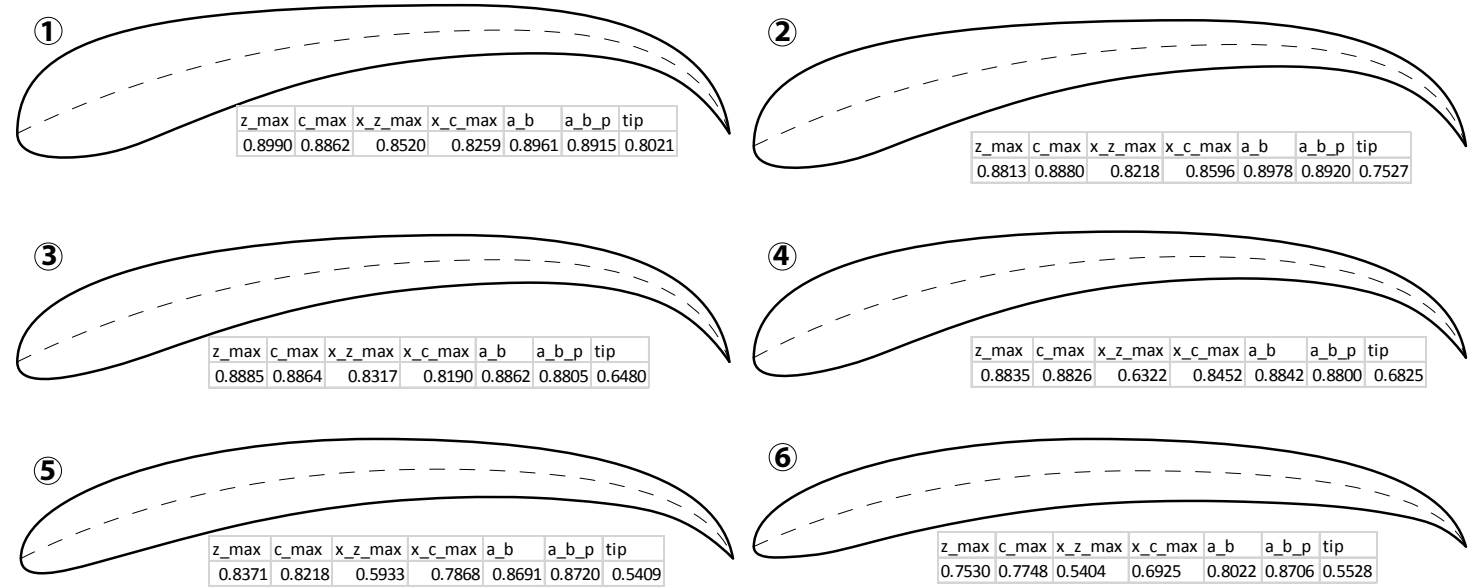

Figure 11: Instances of the hydrofoil parametric model on the Pareto front depicted in Figure 10. Decreasing lift coefficient in a left-to-right, top-to-bottom fashion. 
modeler for the hydrofoil, an Isogeometric BEM solver for the potential flow and an optimizer employing a controlled elitist genetic algorithm. This procedure has been successfully applied for the multi-objective shape optimization of a hydrofoil with respect to the criteria of maximum lift coefficient and minimum deviation of the hydrofoil area from a reference area. The isogeometric BEM solver developed for the steady lifting flow around hydrofoils is robust and effective since it needs much less degrees of freedom for the same accuracy, compared to classical panel methods.

Further work is needed for the improvement of the rate of convergence of the solution by adopting adaptive refinement strategies as well as by treating properly the Boundary Integral Equation near the trailing edge in order to take into account both the corner point of the body boundary and the existence of the wake.

As future work, the extension of the present methodology to 3D problems seems to be straightforward. Moreover, the adoption of boundary-layer corrections to the potential flow should enrich the obtained solutions, from the physical point of view. More challenging, seems to be the problem of shape optimization of 3D hydrofoils moving near the free surface since such systems face major difficulties due to the simultaneous presence of the free surface and the free-vortex systems generated by the lifting bodies which can lead to non-linear phenomena, as, e.g., wave breaking.

\section{References}

[1] T. Hughes, J. Cottrell, Y. Bazilevs, Isogeometric analysis: CAD, finite elements, NURBS, exact geometry and mesh refinement, Computer Methods in Applied Mechanics and Engineering 194 (2005) 4135-4195.

[2] J. A. Cottrell, T. J. R. Hughes, Y. Bazilevs, Isogeometric Analysis: Toward Integration of CAD and FEA, Wiley, 2009.

[3] W. Wall, M. Frenzel, C. Cyron, Isogeometric structural shape optimization, Computer Methods in Applied Mechanics and Engineering 197 (33-40) (2008) 2976-2988.

[4] A. P. Nagy, M. M. Abdalla, Z. Grdal, Isogeometric sizing and shape optimization of beam structures, in: 50th AIAA/ASME/ASCE/AHS/ASC Structures, Structural Dynamics, and Materials Conference, Palm Springs, California, USA, 2009.

[5] D. Nguyen, A. Evgrafov, J. Gravesen, Isogeometric shape optimization for electromagnetic scattering problems, Progress In Electromagnetics Research 45 (2012) 117-146.

[6] P. Nørtoft, J. Gravesen, Isogeometric shape optimization in fluid mechanics, Structural and Multidisciplinary Optimization 48 (2013) 909-925.

[7] X. Qian, O. Sigmund, Isogeometric Shape Optimization of photonic crystals via Coons patches, Computer Methods in Applied Mechanics and Engineering 200 (2011) 2237-2255.

[8] S. Cho, S. Ha, Isogeometric shape design optimization:exact geometry and enhanced sensitivity, Structural and Multidisciplinary Optimization 38 (1) (2009) 53-70.

[9] H. Lian, R. Simpson, S. Bordas, Sensitivity Analysis and Shape Optimisation through a T-spline Isogeometric Boundary Element Method, in: International Conference on Computational Mechanics (CM13), Durham, UK, 2013.

[10] X. Qian, Full analytical sensitivities in NURBS based isogeometric shape optimization, Computer Methods in Applied Mechanics and Engineering 199 (29-32) (2010) 2059-2071.

[11] K. Li, X. Qian, Isogeometric Analysis and Shape Optimization via Boundary Integral, Computer Aided Design 43 (11) (2011) 1427-1437.

[12] K. V. Kostas, A. I. Ginnis, C. G. Politis, P. D. Kaklis, Ship-hull shape optimization with a T-spline based BEM-isogeometric solver, Computer Methods in Applied Mechanics and Engineering 284 (2015) 611-622. 
[13] A. I. Ginnis, R. Duvigneau, C. Politis, K. V. Kostas, K. Belibassakis, T. Gerostathis, P. D. Kaklis, A Multi-Objective Optimization Environment for Ship-Hull Design based on a BEMIsogeometric Solver, in: The fifth Conference on Computational Methods in Marine Engineering (Marine 2013), Hamburg, Germany, 2013.

[14] L. Morino, Boundary Integral Equations in Aerodynamics, Applied Mechanics Reviews 46 (8) (1993) 445-466.

[15] M. Gennaretti, G. Calcagno, A. Zamboni, L. Morino, A high order boundary element formulation for potential incompressible aerodynamics, Aeronautical Journal 102 (1014) (1998) $211-219$.

[16] J. Katz, A. Plotkin, Low Speed Aerodynamics, McGraw Hill, 2001.

[17] F. Paris, J. Canas, Boundary Element Method: Fundamentals and Applications, Oxford University Press, 1997.

[18] L. Dragos, Mathematical Methods in Aerodynamics, Kluwer, 2003.

[19] J. Hess, A. Smith, Calculation of non-lifting potential flow about arbitrary 3-D bodies, Tech. Rep. Report E.S. 40622, Douglas Aircraft (1962).

[20] J. Hess, Calculation of potential flow around arbitrary three dimensional lifting bodies, Tech. Rep. MDC J5679-01, McDonnell Douglas Corporation (1972).

[21] J. Hess, W. Valarezo, Calculation of steady flow around propellers by means of a surface panel method, in: 23rd Aerospace Sciences Meeting, AIAA, Reno, Nevada, 1985.

[22] L. Morino, C.-C. Kuo, Subsonic potential aerodynamics for complex configurations - A general theory, AIAA Journal 12 (2) (1974) 191-197.

[23] S. Kinnas, Theory and numerical methods of hydrodynamic analysis of marine propellers, in: M. Ohkusu (Ed.), Advances in Marine Hydrodynamics, vol. 5, Computational Mechanics Publications, 1996.

[24] K. Belibassakis, G. Politis, A Boundary Integral Equation Formulation of the Neumann Problem for a Vector Field in $\mathbb{R}^{3}$ with Application to Potential Lifting Flows, Engineering Analysis with Boundary Elements 16 (1) (1995) 5-17.

[25] K. Belibassakis, G. Politis, A non-linear velocity based Boundary Element Method for the analysis of marine propellers in unsteady flow, International Shipbuilding Progress 45 (1998) 93-133.

[26] C. Lee, J. Kerwin, A B-spline higher-order panel method applied to two-dimensional lifting problem, Journal of Ship Research 47 (4) (2003) 290-298.

[27] G. Kim, C. Lee, J. Kerwin, A B-spline based higher-order panel method for the analysis of steady flow around marine propellers, Ocean Engineering 34 (2007) 2045-2060.

[28] Z. Gao, Z. Zou, A NURBS based high-order panel method for three-dimensional radiation and diffraction problems with forward speed, Ocean Engineering 35 (2008) 1271-1282.

[29] C. Politis, A. Ginnis, P. Kaklis, K. Belibassakis, C. Feuer, An Isogeometric BEM for exterior potential-flow problems in the plane, in: Proceedings of SIAM/ACM Joint Conference on Geometric and Physical Modeling, California, USA, 2009.

[30] R. Simpson, S. Bordas, J. Trevelyan, T. Rabczuk, A two-dimensional Isogeometric Boundary Element Method for elastostatic analysis, Computer Methods in Applied Mechanics and Engineering 209-212 (2012) 87-100. 
[31] M. Scott, R. Simpson, J. Evans, S. Lipton, S. Bordas, T. Hughes, T. Sederberg, Isogeometric boundary element analysis using unstructured T-splines, Computer Methods in Applied Mechanics and Engineering 254 (2013) 197 - 221. doi:http://dx.doi.org/10.1016/j.cma.2012.11.001.

URL http://www.sciencedirect.com/science/article/pii/S0045782512003386

[32] K. A. Belibassakis, T. P. Gerosthathis, K. V. Kostas, C. G. Politis, P. D. Kaklis, A.-A. Ginnis, C. Feurer, A BEM-Isogeometric method for the ship wave-resistance problem, Ocean Engineering 60 (2013) 53-67.

[33] M. Peake, J. Trevelyan, G. Coates, Extended isogeometric boundary element method (XIBEM) for two-dimensional Helmholtz problems, Computer Methods in Applied Mechanics and Engineering 259 (2013) 93-102.

[34] R. N. Simpson, M. A. Scott, M. Taus, D. C. Thomas, H. Lian, Acoustic isogeometric boundary element analysis, Computer Methods in Applied Mechanics and Engineering 269 (2014) 265290.

[35] A. I. Ginnis, K. V. Kostas, C. G. Politis, P. D. Kaklis, K. A. Belibassakis, T. P. Gerostathis, M. A. Scott, T. J. R. Hughes, Isogeometric Boundary-Element Analysis for the WaveResistance Problem using T-splines, Computer Methods in Applied Mechanics and Engineering 279 (2014) 425-439.

[36] R. Meyer, Introduction to Mathematical Fluid Dynamics, Dover Publications, Inc., 1971.

[37] P. Bassanini, C. Casciola, M. Lancia, R. Piva, A Boundary Integral Equation Formulation of the Neumann Problem for a Vector Field in $\mathbb{R}^{3}$ with Application to Potential Lifting Flows, Meccanica 34 (1) (1999) 199-229.

[38] L. Morino, G. Bernardini, Singularities in BIEs for the Laplace equation: Joukowski trailingedge conjecture revisited, Engineering Analysis with Boundary Elements 25 (2001) 805-818.

[39] L. Piegl, W. Tiller, The Nurbs Book, 2nd Edition, Springer Verlag, 1997.

[40] J. Moran, An introduction to theoretical and computational aerodynamics, J. Wiley \& Sons, 1984 .

[41] K. Deb, Multi-Objective Optimization Using Evolutionary Algorithms, John Wiley \& Sons, 2001. 